\title{
The Elusive Appearance of Hyperenhancing Solid Serous Cystadenoma of the Pancreas-Radio-Pathological Correlation
}

\author{
Supraja Laguduva Mohan ${ }^{1}$ Sunitha Vellathussery Chakkalakkoombil ${ }^{1} \quad$ Sunil Kumar Devaraj ${ }^{1}$ \\ Ravindar Kashyap ${ }^{1}$ Rajesh Nachiappa Ganesh ${ }^{2}$
}

${ }^{1}$ Department of Radiodiagnosis, Jawaharlal Institute of Postgraduate Medical Education and Research (JIPMER), Puducherry, India

2Department of Pathology, Jawaharlal Institute of Postgraduate Medical Education and Research (JIPMER), Puducherry, India

| Gastrointestinal Abdominal Radiol ISGAR 2021;4:77-82.

\begin{abstract}
Address for correspondence Sunitha Vellathussery Chakkalakkoombil, DMRD, DNB, Department of Radiodiagnosis, Jawaharlal Institute of Postgraduate Medical Education and Research (JIPMER),

Puducherry 605006, India (e-mail: drsunithapnair@rediffmail.com).
\end{abstract}

\begin{abstract}
Serous cystadenomas account for $~ 10$ to $29 \%$ of pancreatic cystic tumors. Solid serous cystadenoma (SSCA) is a rare variant, first described in 1996, with imaging characteristics different from the classical serous cystadenoma of the pancreas and can cause a diagnostic dilemma due to its resemblance to other solid tumors of the pancreas. To the best of our knowledge, only 22 cases of SSCA of pancreas have been reported till date. A 50-year-old female patient underwent contrast-enhanced computed tomography (CECT) of the abdomen for a hypoechoic lesion detected in the body of the pancreas during ultrasound (US) examination. Due to the hyperenhancement of the well-circumscribed lesion in the arterial phase, a provisional diagnosis of neuroendocrine tumor was considered. Gallium 68-labeled somatostatin-analog (Ga 68-DOTANOC) positron emission tomography $\mathrm{CT}$ scan did not show any uptake within the lesion and endoscopic US (EUS)-guided fine-needle aspiration cytology (FNAC) was also incon-

Keywords

- solid serous cystadenoma

- hyperenhancing pancreatic lesion

- solid appearing pancreatic lesion clusive. She underwent laparotomy and the lesion was enucleated and it was proven to be a serous microcystadenoma on postoperative histopathologic examination. A diagnosis of SSCA should be considered for solid-appearing pancreatic lesions with characteristic CECT features such as arterial phase hyperenhancement, and immediate washout, along with negative results on DOTANOC scan and EUS-guided FNAC. Malignant transformation of SSCA has not been reported till date, and hence these can be safely followed up, instead of invasive surgery.
\end{abstract}

\section{Introduction}

Cystic pancreatic tumors account for 1 to $2 \%$ of all the exocrine pancreatic neoplasms. ${ }^{1}$ They include benign serous cystadenoma, the potentially malignant mucinous cystadenoma, intraductal papillary cystic neoplasm, solid papillary epithelial neoplasm, and cystic degeneration of solid tumors such as adenocarcinoma and islet cell tumors., ${ }^{1,2}$ Serous cystadenomas can have mainly two types of morphological appearances-microcystic and macrocystic. However, atypical appearances such as solid nature, intratumoral hemorrhage, and large tumor size with ductal dilatation have also been reported in the literature. ${ }^{1}$ We describe the case of a middle-aged woman who presented with a hyperenhancing pancreatic lesion resembling a neuroendocrine tumor (NET) published online

February 1, 2021
DOI https://doi.org/

$10.1055 / \mathrm{s}-0040-1721293$ ISSN 2581-9933.
(C2021. Indian Society of Gastrointestinal and Abdominal Radiology. This is an open access article published by Thieme under the terms of the Creative Commons Attribution-NonDerivative-NonCommercial-License, permitting copying and reproduction so long as the original work is given appropriate credit. Contents may not be used for commercial purposes, or adapted, remixed, transformed or built upon. (https://creativecommons.org/licenses/by-nc-nd/4.0/).

Thieme Medical and Scientific Publishers Pvt. Ltd. A-12, 2nd Floor, Sector 2, Noida-201301 UP, India 
in contrast-enhanced computed tomography (CECT) that was proven to be a solid serous cystadenoma (SSCA) on postoperative histopathologic examination (HPE).

\section{Case Report}

A 50-year-old female presented with complaints of persistent, abdominal pain predominantly in the periumbilical region for the past 3 years. Ultrasound (US) examination of abdomen was done with Esaote MyLab 50 US machine (Esaote, Ahmedabad, Gujarat, India), which showed a hypoechoic lesion in the body of the pancreas, with internal vascularity and with a few calcific specks adjacent to it in the pancreatic parenchyma. Triple-phase CECT of abdomen was done, which included late arterial, portal and hepatic venous phases at 20,50, and 65 seconds, respectively, after injection of $80 \mathrm{~mL}$ of iohexol using Siemens SOMATOM Definition Edge 128 single source dual energy helical CT scanner (Siemens Healthcare $\mathrm{GmbH}$, Erlangen, Germany). A rounded homogeneous hypodense lesion devoid of any calcifications was noted, with an average attenuation of 25 Hounsfield units (HU), in the unenhanced phase in the body of the pancreas (-Fig. 1), which appeared homogeneously hyperenhancing in the arterial phase with mean attenuation of $188 \mathrm{HU}$. There was immediate washout after the arterial phase, with the attenuation decreasing to $90 \mathrm{HU}$ in the portal phase and $87 \mathrm{HU}$ in the hepatic venous phases (-Figs. 2 and 3), whereas the attenuation of adjacent normal pancreatic parenchyma was $\sim 80 \mathrm{HU}$ in the hepatic venous phase. In the portal and hepatic venous phases, the enhancement was more in the periphery of the lesion compared with its center. Foci of calcifications were noted in the pancreas separately from the lesion with focal dilatation of the main pancreatic duct in that region. The hyperenhancing nature of the lesion led to the suspicion of a NET. A subsequent gallium 68 DOTANOC positron emission tomography (PET) CT scan to look for a NET showed no uptake in the lesion, though the possibility of a nonfunctioning NET could still not be ruled out. Endoscopic ultrasound (EUS)-guided fine-needle aspiration cytology (FNAC) also failed to conclude the diagnosis. The patient underwent surgery and enucleation of the tumor was done. HPE showed multiple microcysts within the lesion lined by cuboidal epithelium, with a few hyalinized septae in
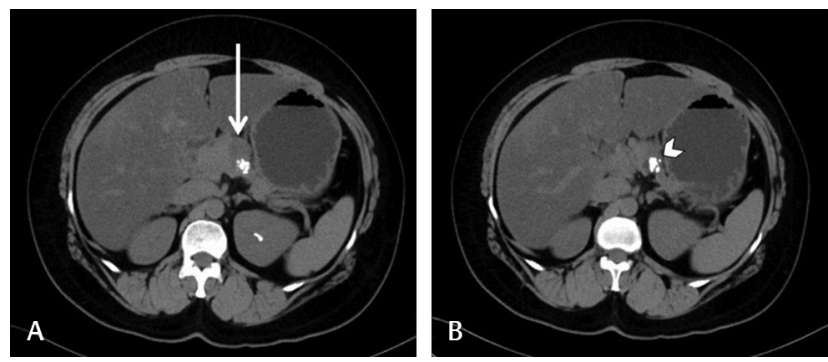

Fig. 1 Plain computed tomography of abdomen showing (A) rounded homogeneous hypodense lesion (arrow) in the proximal part of body of pancreas and (B) focal coarse calcifications separate from the lesion (arrowhead).
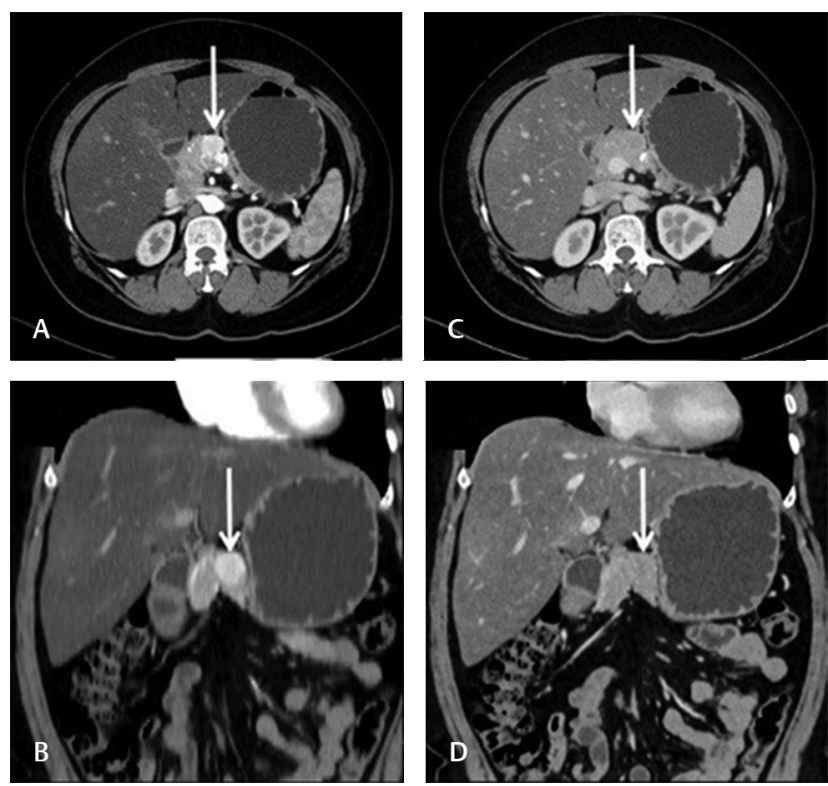

Fig. 2 Contrast-enhanced computed tomography of abdomen: (A, B) axial and coronal images in the arterial phase showing homogeneously hyperenhancing lesion (arrow) in the proximal part of body of pancreas and (C, D) axial and coronal images in the portal phase showing rapid washout of contrast.
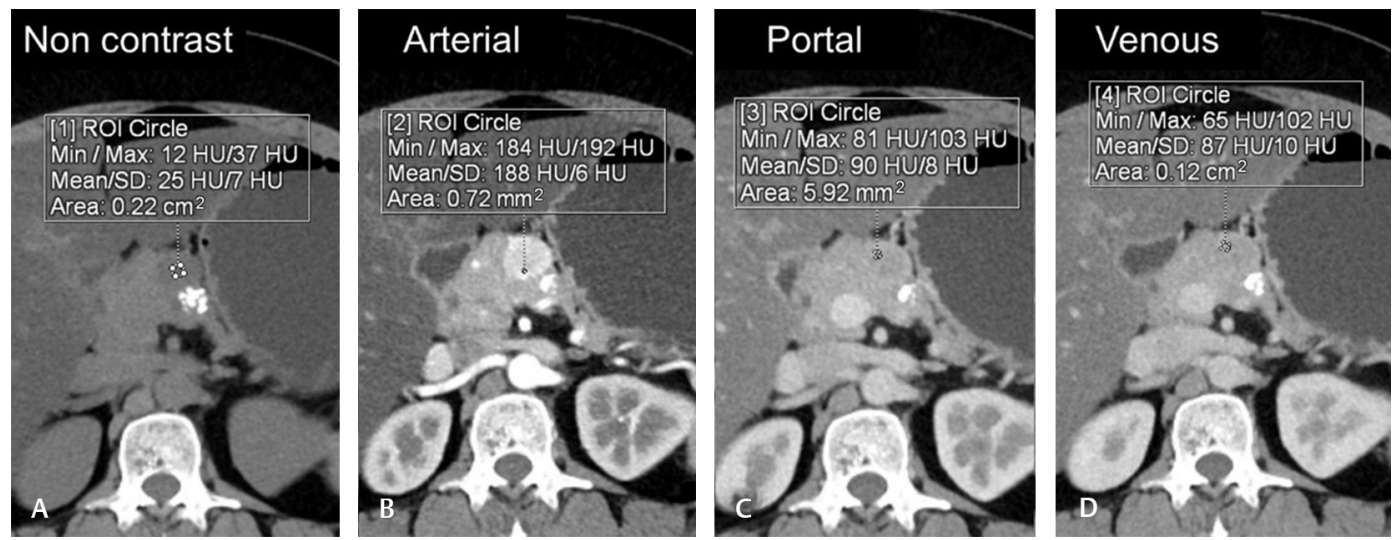

Fig. 3 Enhancement pattern of the lesion in multiphase contrast-enhanced computed tomography (A) Attenuation of 25 Hounsfield unit (HU) in plain computed tomography. (B) The lesion is hyperenhancing, with maximal enhancement in arterial phase with mean attenuation of $188 \mathrm{HU}$ (C) followed by immediate washout and minimal delayed enhancement of $90 \mathrm{HU}$ in the portal phase and (D) $87 \mathrm{HU}$ in the hepatic venous phase. 
between, without any atypia or necrosis and was proven to be a benign SSCA ( - Fig. 4). Postoperative CT done 5 months later showed no residual lesion

\section{Discussion}

About 10 to $29 \%$ of cystic tumors of the pancreas are serous cystadenomas. They can be the more common microcystic type that accounts for $90 \%$ of the cases or the less common oligocystic/macrocystic type. ${ }^{3,4}$ Solid forms of serous cystadenomas are extremely rare. It was first described in 1996 as a solid tumor that contained cells resembling those in serous cystadenoma and was suggested to be its variant. ${ }^{4}$ Till now, as per the available literature only around 22 cases of SSCA have been reported, among which seven cases had an appearance similar to that of the current case, with homogeneous hyperenhancement in the arterial phase., ${ }^{4,5}$

Histologically, serous cystadenomas are composed of cystic spaces lined by cuboidal cells representing pancreatic ductal epithelium, rich in glycogen with oval to round nuclei. The lumen of the cysts is filled with clear fluid; however, in $45 \%$ of cases, there can be hemorrhage into the cyst lumen. The more the cysts are distended with fluid, the more flattened is the epithelium lining them. Necrotic areas, infiltrative architecture, or mitotic figures will be absent as they have no malignant potential unlike mucinous cystadenomas or intraductal papillary mucinous neoplasms. In the microcystic variant, there are thin-walled cysts with small intervening septae containing a network of subepithelial vessels (-Fig. 5). The macrocystic type has larger cysts and can be unilocular in $50 \%$ of the cases. In the solid variant, there are no cysts, but they have been described to contain small nests or tubules with minimal lumen formation. There is also a mixed serous neuroendocrine variant with a well-differentiated component of NET in addition to the serous component. ${ }^{6}$

SSCAs are usually smaller in size, measuring $\sim 2.5$ to $4 \mathrm{~cm}$. They are well-defined masses that do not show any invasion into adjacent structures. The cystic acinar spaces are small and are separated by trabeculae made up of fibrocollagenous tissue with rich blood supply ( - Fig. 5). The radiological appearance is usually confused with NET, solid pseudopapillary neoplasms
(SPNs), and pancreatic metastasis from renal cell carcinoma (-Table 1). The previously reported lesions are hypoechoic on US and most of them have shown arterial hyperenhancement in CECT in the early phases with washout, due to the rich vascularity and weak central enhancement in the delayed phases attributed to the fibrous trabeculae within it. ${ }^{7}$ It has also been reported that the washout rates of SSCA are higher than that of NETs. On magnetic resonance imaging, they tend to show a high T2 signal intensity, higher than that of NETs. Usually adequate sampling of these lesions by EUS-guided FNAC cannot be achieved, unlike other solid pancreatic neoplasms; hence, it often fails to conclude the diagnosis. ${ }^{5,7}$ However, when sufficient sample is obtained it helps to confirm the diagnosis or rule out other solid neoplasms of pancreas.
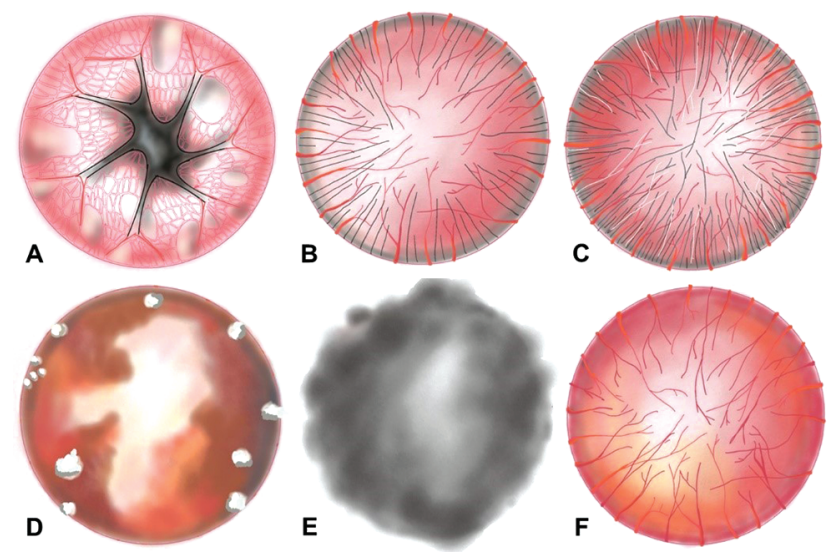

Fig. 5 Histological and macroscopic features of solid serous cystadenoma and its differentials that determine their imaging appearances. (A) Typical serous cystadenoma-multiple small cystic spaces, trabeculae, and central scar (delayed enhancement of central scar). (B) Solid serous cystadenoma-very tiny cysts creating a "solid" appearance, rich blood supply along fibrocollagenous trabeculae (hyperenhancing with immediate washout, mild delayed enhancement). (C) Similar appearance in neuroendocrine tumors, but with more fibrocollagenous stromacontributing to their lower washout rate. (D) Solid pseudopapillary neoplasm-cystic and enhancing components, with peripheral calcifications. (E) Pancreatic adenocarcinoma-irregular margins and rich in fibrous tissue with desmoplastic reaction (hypoenhancing, delayed enhancement). (F) Hypervascular metastasis-most commonly from renal cell carcinoma, rich vascularity leading to hyperenhancement in arterial phase with washout (Image Courtesy: Dr. Supraja L.M.).
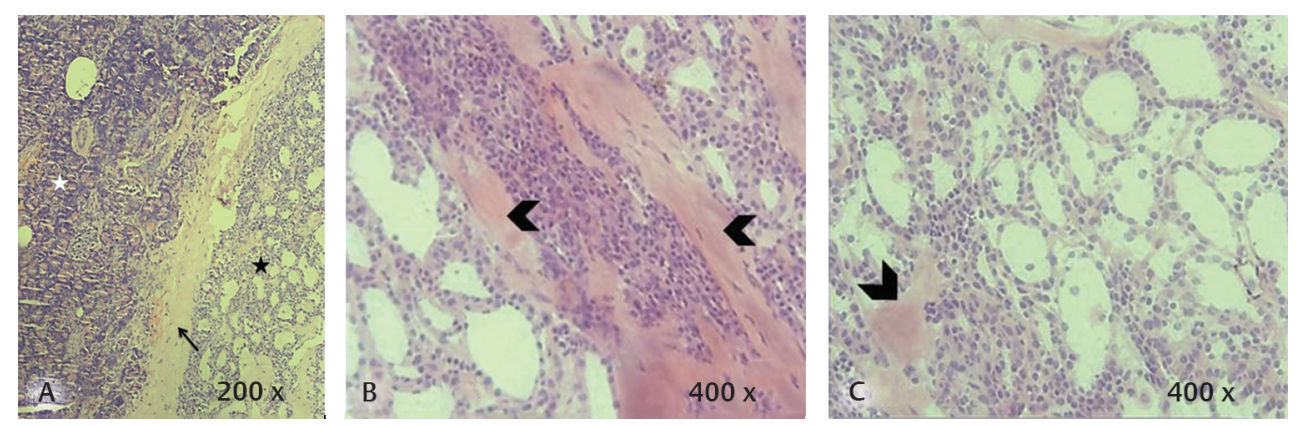

Fig. 4 Histopathology images: (A) The lesion with multiple small cystic spaces (black asterisk) surrounded by a capsule (black arrow). White asterisk depicts the surrounding normal pancreatic parenchyma. (B) Proper tumor with variably sized cystic spaces lined by low cuboidal epithelium. Few areas of hyalinized septa are noted in between cystic spaces (black arrowheads). (C) Higher magnification highlighting the bland morphology of tumor cells. 
Table 1 Solid tumors of the pancreas-Clinical, pathological, and imaging characteristics ${ }^{10-15}$

\begin{tabular}{|l|l|l|l|l|l|}
\hline Characteristics & SSCA & NET & SPN & $\begin{array}{l}\text { Hypervascular } \\
\text { metastasis }\end{array}$ & Adenocarcinoma \\
\hline Age & Elderly (female) & 4th to 6th decade & Young female & Elderly & 7th decade \\
\hline $\begin{array}{l}\text { Common tumor } \\
\text { location in the } \\
\text { pancreas }\end{array}$ & Head & Varies with the type & Tail & $\begin{array}{l}\text { Evenly } \\
\text { throughout } \\
\text { pancreas }\end{array}$ & $\begin{array}{l}\text { Head and uncinate } \\
\text { process }\end{array}$ \\
\hline $\begin{array}{l}\text { Significant } \\
\text { pathological } \\
\text { features }\end{array}$ & $\begin{array}{l}\text { Very tiny cysts } \\
\text { causing a solid } \\
\text { appearance, rich } \\
\text { vascularity through } \\
\text { fibrocollagenous } \\
\text { trabeculae }\end{array}$ & $\begin{array}{l}\text { Rich fibrocollagenous } \\
\text { stroma and vascularity }\end{array}$ & $\begin{array}{l}\text { Cystic and solid } \\
\text { components, } \\
\text { peripheral calcifications }\end{array}$ & $\begin{array}{l}\text { Rich vascularity } \\
\text { stroma }\end{array}$ & Dense fibroblastic \\
\hline Arterial phase & Hyperenhancing & $\begin{array}{l}\text { Hyperenhancing; } \\
\text { larger tumors can be } \\
\text { heterogeneous }\end{array}$ & $\begin{array}{l}\text { Mild enhancement of } \\
\text { solid component }\end{array}$ & Hyperenhancing & Hypoenhancing \\
\hline $\begin{array}{l}\text { Portal/venous } \\
\text { phase }\end{array}$ & Washout & Delayed washout & $\begin{array}{l}\text { Heterogeneous } \\
\text { enhancement }\end{array}$ & Washout & $\begin{array}{l}\text { Delayed } \\
\text { enhancement }\end{array}$ \\
\hline Prognosis & Good & Intermediate & Poor & Poor \\
\hline
\end{tabular}

Abbreviations: NET, neuroendocrine tumor; SPN, solid pseudopapillary neoplasm; SSCA, solid serous cystadenoma;

ansulinoma and nonfunctioning tumors are evenly distributed throughout the pancreas; gastrinoma and somatostatinoma in the head; VIPoma and glucagonoma in the tail.

Hayashi et al have described the differences in dynamic CECT between NETs of the pancreas and SSCAs. ${ }^{9}$ SSCAs are more frequently hypodense on plain sections than NETs, explained by the fact that SSCA are still microcystic and made up of numerous small "cysts" compared with the completely solid NETs. The washout rate is higher in SSCA than in NET, because of the increased fibrocollagenous stroma in NET. Central scars that are classic of serous cystadenomas are rare in the solid variant-however, a fibrous capsule is seen more frequently in SSCA than in NET. The capsule shows delayed enhancement. ${ }^{8,9}$ In our case, the lesion was hypodense on plain sections and showed arterial phase hyperenhancement and significant washout, with minimal delayed enhancement. This minimal enhancement in the delayed phase can be reasoned by the presence of collagenous septae within the lesion, which was seen microscopically.

Out of the previously described 22 cases, most of the lesions were either in the head or body (15 out of 22 cases). Almost half of them were incidentally detected, whereas in rest of the lesions, patients presented with abdominal pain or weight loss. The lesions were encapsulated, small size in size, and most of the patients belonged to the age group of 55 to 70 years. The most common presumed diagnosis before surgery was a NET. Others were solid pseudopapillary neoplasm, metastasis, and adenocarcinoma. Seven of the previously reported cases had enhancement pattern similar to our case with complete homogeneous hyperenhancement, whereas the rest of them showed peripheral hyperenhancement with central nonenhancing areas or minimal peripheral enhancement. ${ }^{10}$

Pancreatic metastasis, particularly from hypervascular primaries such as kidney and thyroid, may also show hyperenhancement in the arterial phase and washout. However, these lesions are very rare and are found in the advanced stages of malignancy. There is no predilection for the location of these lesions to the pancreas and they can be any of the three types-(1) solitary circumscribed mass, (2) diffusely enlarged pancreas, and (3) multiple nodules. ${ }^{11}$ Pancreatic adenocarcinomas, on the other hand, appear hypoenhancing to the rest of the pancreas in the arterial phase and enhance in the delayed phase due to their dense fibroblastic stroma ( $\mathbf{- F i g . 5 E}$ ). They can also lead to alteration of the contour of the pancreas, with abrupt tapering of the main pancreatic duct or common bile duct or both. Most of these lesions occur in the head or uncinate process of the pancreas. ${ }^{12}$ SPNs are large well-encapsulated masses that occur more commonly in the pancreatic tail, more commonly in young females. They consist of cystic and enhancing solid components, due to hemorrhagic degeneration ( - Fig. 5D). Peripheral calcifications can be seen. The two most important features pointing toward SPN are the capsule and intralesional hemorrhage, as these features are rarely seen in other pancreatic neoplasms. ${ }^{13-15}$

To conclude, SSCA should be considered as a possible differential diagnosis when a well-defined solid-appearing pancreatic lesion, showing arterial phase hyperenhancement, high washout ratio, and persistent delayed enhancement, is seen in CECT that fails to get characterized on DOTANOC PET CT scan. EUS-guided FNAC will help either to confirm SSCA or to rule out other pancreatic solid neoplasms. Malignant transformation of SSCA has not been reported previously. Hence, once a lesion is confirmed as SSCA by imaging and tissue diagnosis, it can be followed up carefully, instead of invasive surgical interventions. ${ }^{8}$

\section{Funding}

None.

\section{Conflict of Interest}

None declared. 


\section{References}

1 Choi J-Y, Kim M-J, Lee JY, et al. Typical and atypical manifestations of serous cystadenoma of the pancreas: imaging findings with pathologic correlation. AJR Am J Roentgenol 2009;193(1):136-142

2 Khan A, Khosa F, Eisenberg RL. Cystic lesions of the pancreas. AJR Am J Roentgenol 2011;196(6):W668-W677

3 Patient Care Guidelines SS; Society for Surgery of the Alimentary Tract. SSAT patient care guidelines. Cystic neoplasms of the pancreas. J Gastrointest Surg 2007;11(9):1225-1227

4 Yamaguchi M. Solid serous adenoma of the pancreas: a solid variant of serous cystadenoma or a separate disease entity? J Gastroenterol 2006;41(2):178-179

5 Kishida Y, Matsubayashi H, Okamura Y, et al. A case of solid-type serous cystadenoma mimicking neuroendocrine tumor of the pancreas. J Dig Dis 2014;15(4):211-215

6 Charville GW, Kao C-S. Serous neoplasms of the pancreas: a comprehensive review. Arch Pathol Lab Med 2018; 142(9):1134-1140

7 Katsourakis A, Dimitriou I, Noussios G, Chatzis I, Chatzitheoclitos E. Solid Serous Adenoma of the Pancreas: A Case Report and Review of the Literature. Case Rep Surg; 2016; 2016:3730249
8 Machado MC, Machado MA. Solid serous adenoma of the pancreas: an uncommon but important entity. Eur J Surg Oncol 2008;34(7):730-733

9 Hayashi K, Fujimitsu R, Ida M, et al. CT differentiation of solid serous cystadenoma vs endocrine tumor of the pancreas. Eur J Radiol 2012;81(3):e203-e208

10 Demesmaker V, Abou-Messaoud F, Parent M, Vanhoute B, Maassarani F, Kothonidis K. Pancreatic solid serous cystadenoma: a rare entity that can lead to a futile surgery. J Surg Case Rep 2019;2019(12):rjz360

11 Scatarige JC, Horton KM, Sheth S, Fishman EK. Pancreatic parenchymal metastases: observations on helical CT. AJR Am J Roentgenol 2001;176(3):695-699

12 Bronstein YL, Loyer EM, Kaur H, et al. Detection of small pancreatic tumors with multiphasic helical CT. AJR Am J Roentgenol 2004;182(3):619-623

13 Choi J-Y, Kim M-J, Kim JH, et al. Solid pseudopapillary tumor of the pancreas: typical and atypical manifestations. AJR Am J Roentgenol 2006;187(2):W178-W186

14 BalciNC,Semelka RC. Radiologic diagnosis and staging of pancreatic ductal adenocarcinoma. Eur J Radiol 2001;38(2):105-112

15 Lewis RB, Lattin GE Jr, Paal E. Pancreatic endocrine tumors: radiologic-clinicopathologic correlation. Radiographics 2010; 30(6):1445-1464 